\title{
Rare RNF213 variants and the risk of intracranial artery stenosis/occlusion disease in Chinese population: a case- control study
}

\author{
Xin Liao ${ }^{1 \dagger}$, Tong Zhang ${ }^{2 \dagger}$, Bingyang $\mathrm{Li}^{1}$, Shimin Hu${ }^{1}$, Junyu Liư ${ }^{3}$ Jing Deng ${ }^{1}$, Hongzhuan $\operatorname{Tan}^{1}$ and Junxia Yan ${ }^{1^{*}}$ (D)
}

\begin{abstract}
Background: RNF213 rare variant-p.R4810K (rs112735431) was significantly associated with intracranial artery stenosis/occlusion disease (ICASO) in Japan and Korea and to a lesser degree in China. Considering the allelic heterogeneity, we performed target exome sequencing of RNF213 with the aim to identify the rare variants spectrum and their association with ICASO in a Chinese population and further to explore whether the rare variants carrier patients present specific clinical phenotype.

Methods: Target exome sequencing of RNF213 was performed in 250 ICASO patients using FastTarget sequencing technology. Various filtering process were used to select the candidate variants. Control individuals were obtain from 1000 Genome Project (208 Chinese samples) and GeneSky in-house database (1007 samples). Gene-based association analyses were conducted to identify the association between RNF213 rare variants and ICASO. The clinical characteristics of rare variant carriers and non-carriers were compared using Chi-squared test or Fisher's exact test.

Results: After filtration, 18 rare variants were identified in 39 patients. Gene-based association test showed that rare variants of RNF213 were significantly associated with ICASO (Minor allele frequency $<0.05$, WSS $p=4.88 \times 10^{-10}$; SKAT $p=9.68 \times 10^{-6}$; SKAT-O $\left.p=3.42 \times 10^{-9}\right)$. There were no significant clinical characteristic differences other than the diagnosis age which was older in the carriers than the non-carriers ( $60.5 \pm 6.2$ vs $57.3 \pm 8.9$ years old, $p=0.028$ ).
\end{abstract}

Conclusion: Rare variants of RNF213 are associated with ICASO in Chinese. However, there are limited genetic diagnosis values of the gene due to no specific phenotypic presentation in the carriers and non carrier patients.

Keywords: RNF213, Intracranial artery stenosis, Rare variants

\section{Background}

Intracranial artery stenosis/occlusion disease (ICASO) is one of the main causes of ischemic stroke [1]. It is more prevalent in Asians than in Westerners. In Asia, $30-50 \%$ of strokes and more than $50 \%$ of transient ischemic attacks are caused by ICASO [2]. Atherosclerosis, chronic injury, infection, cardioembolism, arterial dissection, vasculitis, moyamoya disease (MMD) and rare autoimmune diseases are common cause of ICASO

\footnotetext{
* Correspondence: 20457456@qq.com

${ }^{+}$Xin Liao and Tong Zhang contributed equally to this work.

${ }^{1}$ Department of Epidemiology and Health Statistics, XiangYa School of Public Health, Central South University, Changsha 410078, Hunan, China

Full list of author information is available at the end of the article
}

[3]. The most common cause of ICASO is atherosclerosis, which is commonly caused by acquired factors, such as smoking, hypertension, diabetes mellitus and dyslipidemia [3]. However, conventional risk factors only explain part of risks and the underlying etiologies is largely unknown. Many studies supported a genetic risk role of ICASO [3-10].

The ring finger protein 213 (RNF213) was found to be a susceptibility gene for MMD in East Asian population and the founder rare variant-p.R4810K (rs112735431) was subsequently identified to be associated with non-MMD ICASO which showed stenosis or occlusion of the intracranial major arteries but do not meet the diagnostic criteria of MMD [3-6, 11-21]. Meta-analysis

(c) The Author(s). 2019 Open Access This article is distributed under the terms of the Creative Commons Attribution 4.0 International License (http://creativecommons.org/licenses/by/4.0/), which permits unrestricted use, distribution, and reproduction in any medium, provided you give appropriate credit to the original author(s) and the source, provide a link to the Creative Commons license, and indicate if changes were made. The Creative Commons Public Domain Dedication waiver (http://creativecommons.org/publicdomain/zero/1.0/) applies to the data made available in this article, unless otherwise stated. 
showed that RNF213 p.R4810K increased the risk of ICASO in Chinese (odds ratio, 5.59; 95\% confidence interval, $2.12-14.75 ; p=0.001$ ), but the effect size was significantly lower than that in Japanese and Korean (odds ratios, 10.71 and 28.52; 95\% confidence interval, 3.97-28.91; and 11.04-73.67, respectively) [22]. Further studies found that many non-p.R4810K RNF213 rare variants have been identified in MMD patients in ethnically diverse populations, including Asians, whites, and Hispanics, while p.R4810K is absent in non-Asian populations, which illustrating allelic genetic heterogeneity of RNF213 in different ethnic population [23]. Whether other RNF213 rare variants contribute to Chinese ICASO is still unknown. Considering the allelic heterogeneity, we performed target exome sequencing of RNF213 with the aim to identify the rare variants spectrum and their association with ICASO in a Chinese population and further to explore whether the rare variants carriers present specific clinical phenotype or not.

\section{Methods \\ Study population}

Seven hundred and fifteen ICASO patients were recruited from two university affiliated hospitals (615 from the Second Hospital of Hebei Medical University and 100 from XiangYa Hospital of Central South University) previously [9]. Among them, only 6 patients carried RNF213 p.P4810K variants. In order to investigate whether other RNF213 rare variants associated with ICASO or not, 250 patients were randomly selected from our previous individuals. Allelic frequencies of control individuals were obtained from 1000 Genome Project Chinese Han Population (208 samples, http://www. internationalgenome.org/home) and GeneSky in-house database (1007 samples, http://www.geneskybiotech. com/index.html). This study was approved by the Medical Ethics Committee of Central South University and all patients signed an informed consent before participating.

\section{Diagnosis of ICASO}

The ICASO was diagnosed by digital subtraction angiography, computed tomography angiography or magnetic resonance angiography and defined as stenosis or occlusion at terminal and/or proximal portions of the intracranial major arteries without abnormal vascular networks in the basal ganglia that did not meet the diagnostic criteria of MMD. At least 2 physicians(1 radiologist and 1 neurological physician) were required to interpret angiography images results. Demographic information including age, gender, behavioral risk factors (tobacco and alcohol consumption), disease histories of hypertension, diabetes, hyperlipemia were collected by interview or questionnaire. Patients with potential diseases including cardioaortic embolism, coagulopathy, vasculitis, arterial dissection that may leads to cerebral apoplexy were excluded.

\section{FastTarget sequencing}

Genomic DNA with high quality suitable for sequencing was extracted from the peripheral blood leukocytes using TIANamp Blood DNA Extraction Kit (TIANGEN BIOTECH CO., LTD, Beijing, China). Protein coding sequences of RNF213 were selected and a custom panel covering the target regions was designed. Capture target regions contained flanking sequences of 25 bases at the borders of each exon. Target sequencing was performed on Illumina Hiseq/MiSeq high-throughput sequencing platform (Illumina, San Diego, CA, USA) according to the manufacturer's instructions. Raw fastQ files were aligned to the human reference sequence (NCBI Genome build GRCh37, https://www.ncbi.nlm.nih.gov/genome/guide/ human/\#download) with the Burrows-Wheeler Aligner (BWA, http://bio-bwa.sourceforge.net/). The HaplotypeCaller from the Genome Analysis Toolkit (GATK, https:// software.broadinstitute.org/gatk/best-practices/) and VarScan (http://varscan.sourceforge.net/) were used for variant calling in the target region. Quality of sequencing was determined with FastQC (http://www.bioinformatics. babraham.ac.uk/projects/fastqc/). PolyPhen-2 (http://gen etics.bwh.harvard.edu/pph2/) and SIFT (http://sift.bii.a-s tar.edu.sg/) were used for predicting the deleterious level of the functional variants.

\section{Variant filtration}

After sequencing, a series of filters were used to prioritize variants. Variants were considered higher priority if they were: (a) predicted to affect protein-coding sequences (including nonsynonymous, frameshift deletion, stop-gain variants in exonic or splicing regions); (b) less common in reference databases (Minor allele frequency was less than 0.05 in 1000 Genome project Chinese Han population [ftp://ftp.1000genomes.ebi.ac. uk/vol1/ftp/release/20130502/] and GeneSky in-house Database [http://www.geneskybiotech.com/]); (c) predicted as damaging, possibly damaging or unknown by SIFT or PolyPhen-2; (d) sequencing read depth was higher than 10x.

\section{Statistical analysis}

Descriptive analyses were conducted using SPSS 21.0 software (SPSS Inc., Chicago, IL, USA). Continuous variable (age) was presented as the mean \pm standard deviation (SD). Categorical variables (hypertension, diabetes, hyperlipemia, smoking, drinking, clinical symptoms and characteristics of stenosis) were presented as proportions. The Weight Sum Statistic (WSS), the Sequence Kernel Association Test (SKAT) and the SKAT-optimal 
test (SKAT-O) were used to evaluated the statistical significance of variants between affected and control individuals. A continuous weight function proposed by Madsen and Browning was performed to focus on the lower frequency and rarer variants, this weight score method allows both low frequency $(\mathrm{MAF}<0.05)$ and rare $(\mathrm{MAF}<0.01)$ variants to contribute to the overall statistic by calculating the logistic weights for each variant and applying them to the analysis according to the following formula [24]: $w_{j}=1 /\left[M A F_{j}\left(1-M A F_{j}\right)\right]^{1 / 2}$. Student's t-test, Chi-squared test or Fisher's exact test were used to compare the characteristic differences between carriers and non-carriers of RNF213 rare variants. Threshold of statistical significance was set at $p$ value less than 0.05 .

\section{Result}

\section{Characteristics of participants}

Of all the 250 patients with ICASO, 77(30.8\%) were female and the average age was $57.8 \pm 8.6$ years (range: 28-70 years). The information of the vascular risk factors, clinical symptoms and characteristics of cerebral artery stenosis were summarized in Table 1.

\section{Identification of low frequency and rare variants in target region}

The average value of mean target coverage was $527 x$, $88.87 \%$ of the reads had a Phred-like quality score (Q-score) greater than 30.The proportion of the samples obtained mean coverage $>140 \mathrm{x}$ was $94.24 \%$. After variants filtration, 18 variants were found in 39 patients (Table 2). The detail information of these rare variants was shown in Table 3.

\section{Distribution of rare variants in ICASO patients and gene- based association test}

Eighteen rare RNF213 variants were found in ICASO including 6 splicing site variants, 11 missenses and 1 frameshift deletion. All the missense variants were predicted to be deleterious, 6 of them were absent from the control individuals, and 6 of them were clustered at the C-terminal domain of RNF213 (exon 42-68) (Table 3, Additional file 1: Figure S1). We identified a total 42 alternative alleles in ICASO patients $(n=250)$ and 117 alternative alleles in controls $(n=1215$, cumulative allele OR, $1.74 ; 95 \% \mathrm{CI}$, $1.23-2.49, p=0.002)$. Single-variant association test revealed that none of the SNPs was associated with ICASO (Table 4). Therefore, we focus on gene-based burden test to evaluate the aggregate effects of low MAF variants. We performed WSS, SKAT, SKAT-O test at two MAF thresholds $(\mathrm{MAF}<0.05,0.01)$ respectively. The WSS and SKAT-O test using logistic weights to compare RNF213 functional variants
Table 1 Characteristics of the case participants and controls ${ }^{d}$

\begin{tabular}{|c|c|}
\hline Characteristics & $\mathrm{ICASO}(n=250)$ \\
\hline \multicolumn{2}{|l|}{ Age(years) } \\
\hline Mean \pm SD [Range] & $57.8 \pm 8.6[28-70]$ \\
\hline Female, $n(\%)$ & 77(30.8) \\
\hline \multicolumn{2}{|l|}{ Risk factors, n(\%) } \\
\hline Smoking & 97(38.8) \\
\hline Drinking & $63(25.2)$ \\
\hline Hypertension & $152(60.8)$ \\
\hline Diabetes & $60(24.0)$ \\
\hline Hyperlipemia & 95(38.0) \\
\hline \multicolumn{2}{|l|}{ Clinical symptoms, n(\%) } \\
\hline Hemorrhage & $7(2.8)$ \\
\hline Infarction & 165(66.0) \\
\hline Ischemia & 77(30.8) \\
\hline Other & $9(3.6)$ \\
\hline Multiple stenosis & 199(79.6) \\
\hline \multicolumn{2}{|l|}{ Location } \\
\hline Anterior circulation $^{a}$ & $36(14.4)$ \\
\hline Posterior circulation ${ }^{\mathrm{b}}$ & 99(39.6) \\
\hline Multiple circulation ${ }^{c}$ & $111(44.4)$ \\
\hline Others & 4(1.6) \\
\hline
\end{tabular}

${ }^{a}$ Anterior circulation $=A C A$ Anterior cerebral artery, MCA Middle cerebral artery, ICA Internal carotid artery

${ }^{\mathrm{b}}$ Posterior circulation = BA Basilar artery, VA Vertebral artery, PCA Posterior cerebral artery

${ }^{c}$ Multiple circulation $=$ including both Anterior and posterior circulation ${ }^{d}$ Control individuals were obtained from 1000 Genome Project Chinese Han Population and GeneSky in-house database, clinical characteristics were not available

$(\mathrm{MAF}<0.05)$ showed significantly different allelic distributions between the ICASO patients and control individuals (WSS $p=4.88 \times 10^{-10}$; SKAT $p=9.68 \times 10^{-6} ; \quad$ SKAT-O $p=3.42 \times 10^{-9}$.). For the rarer RNF213 functional variants $(\mathrm{MAF}<0.01)$, gene-based test showed more significant difference (WSS $\quad p=4.56 \times 10^{-12}$; SKAT $\quad p=2.80 \times 10^{-6}$; SKAT-O $p=3.20 \times 10^{-14}$ ) (Table 2$)$.

\section{Clinical characteristics of patients with and without RNF213 rare variants}

Among the 250 ICASO patients, we found 39 patients carried RNF213 rare variants and compared the clinical characteristics between the carriers and non-carriers. The results showed that patients with RNF213 rare variants were older at diagnosis $(60.5 \pm 6.2$ vs $57.3 \pm 8.9$ years old, $p=0.028$ ). Risk factors of ICASO (including smoking, alcohol drinking, hypertension, diabetes, hyperlipemia), clinical symptoms and characteristics of cerebral artery lesions (quantity of lesions, presence of anterior and posterior ICASO) showed no significant difference between two groups (Table 5). 
Table 2 Variant Filtration Steps of sequencing and results of gene-based analysis

\begin{tabular}{|c|c|c|c|}
\hline Filtration Steps & \multicolumn{3}{|c|}{ Number of variants } \\
\hline 1.SNVs ${ }^{\mathrm{a}}$ located in exonic/ splicing region & \multicolumn{3}{|l|}{188} \\
\hline 2. Nonsynonymous SNVs/ frameshift deletion/ stopgain & \multicolumn{3}{|l|}{125} \\
\hline $\begin{array}{l}\text { 3. Variants judged as SIFT prediction = Damaging / unknown and PolyPhen-2 } \\
\text { prediction = Possibly damaging/ Probably damaging/ Unknown }\end{array}$ & \multicolumn{3}{|l|}{71} \\
\hline 4. MAF $<0.05$ in $1 \mathrm{KGP}($ Chinese Han population) and GeneSky in-house Database & \multicolumn{3}{|l|}{42} \\
\hline 5.Variants with sequenced base depth $>10 x$ & \multicolumn{3}{|l|}{18} \\
\hline \multicolumn{4}{|l|}{ Result of RNF213 gene-based analysis } \\
\hline MAF & Pwss & $P_{\text {SKAT }}$ & $P_{\text {SKAT-O }}$ \\
\hline$<0.05$ & $4.88 \times 10^{-10}$ & $9.68 \times 10^{-6}$ & $3.42 \times 10^{-9}$ \\
\hline$<0.01$ & $4.56 \times 10^{-12}$ & $2.80 \times 10^{-6}$ & $3.20 \times 10^{-1}$ \\
\hline
\end{tabular}

${ }^{\mathrm{a}} \mathrm{SNV}$ s Single Nucleotide Variants

\section{Discussion}

In this study, we sequenced the exome of RNF213 in 250 ICASO patients and identified 18 rare variants in 39 patients. Gene-based association test showed that rare variants of RNF213 were significantly associated with ICASO. However, there were no significant clinical characteristic differences between the rare variants carriers and non-carriers.

RNF213 founder mutation-p.R4810K (rs112735431) was identified to be significantly associated with MMD and non-MMD ICASO in East Asia. Other than
p.R4810K, numerous rare variants also were identified in MMD cases in previous studies. In Japan, Kamada et al. identified 3 non-p.R4810K rare variants of RNF213 (p.M3891 V, p.V4567 M and p.V4765 M) among MMD patients [11]. Subsequently, Moteki et al. found 16 rare coding variants of RNF213 in MMD cases by exome sequencing, including 2 previously identified variants (p.V4567 $\mathrm{M}$ and p.V4765 M) and 14 novel variants (Additional file 1: Figure S1, Additional file 2: Table S1) [25]. In China, researchers identified 40 RNF213 rare variants among MMD patients. All of these variants

Table 3 Rare variants detected in ICASO patients

\begin{tabular}{|c|c|c|c|c|c|c|c|c|c|c|}
\hline \multirow[t]{2}{*}{ Position } & \multirow[t]{2}{*}{ Gene Region } & \multirow[t]{2}{*}{ Function } & \multicolumn{2}{|l|}{ Variant $^{\mathrm{a}}$} & \multirow[t]{2}{*}{ SNP ID } & \multicolumn{3}{|c|}{ Genotype $^{b}$} & \multirow[t]{2}{*}{$\mathrm{SIFT}^{\mathrm{C}}$} & \multirow[t]{2}{*}{ POLYPhen V2 } \\
\hline & & & cDNA & Amino Acid & & Case & 1000G_CHB & GENESKY & & \\
\hline Chr17:78247076-78,247,076 & exonic & frameshift & c.134delC & p.S45 fs & - & $249 / 1 / 0$ & 208/0/0 & $1007 / 0 / 0$ & - & - \\
\hline Chr17:78298891 & exonic & missense & c.3086 T > C & p.L1029S & rs753208141 & $249 / 1 / 0$ & 208/0/0 & $1007 / 0 / 0$ & D & $\mathrm{D}$ \\
\hline Chr17:78307986 & exonic & missense & $c .4225 G>T$ & p.D1409Y & - & $249 / 1 / 0$ & $208 / 0 / 0$ & $1007 / 0 / 0$ & $D$ & D \\
\hline Chr17:78311532 & splicing & - & $c .4668+6 C>T$ & - & rs78795452 & $248 / 2 / 0$ & $205 / 3 / 0$ & 1007/0/0 & - & - \\
\hline Chr17:78311620 & splicing & - & c.4669-13A > G & - & rs750893752 & 249/1/0 & 208/0/0 & $999 / 8 / 0$ & - & - \\
\hline Chr17:78313764 & exonic & missense & c.5597C > T & p.T1866l & rs546687179 & $244 / 6 / 0$ & $207 / 1 / 0$ & $1007 / 0 / 0$ & D & - \\
\hline Chr17:78318465 & splicing & - & c. $6343-13 C>G$ & - & rs141121193 & $246 / 4 / 0$ & $206 / 2 / 0$ & $992 / 15 / 0$ & - & - \\
\hline Chr17:78319385 & exonic & missense & c.7250 T > G & p.l2417s & rs181965032 & $244 / 6 / 0$ & 201/7/0 & $992 / 15 / 0$ & $\mathrm{D}$ & $P$ \\
\hline Chr17:78326772 & exonic & missense & c. $10336 \mathrm{C}>\mathrm{T}$ & p.R3446W & rs776943470 & $249 / 1 / 0$ & 208/0/0 & $992 / 15 / 0$ & D & $\mathrm{D}$ \\
\hline Chr17:78350088 & splicing & - & c.13186-13 T>C & - & rs113236556 & $239 / 11 / 0$ & $202 / 6 / 0$ & 1006/1/0 & - & - \\
\hline Chr17:78353469 & exonic & missense & c. $13595 \mathrm{~T}>\mathrm{C}$ & p.14532T & rs373648166 & 249/1/0 & 208/0/0 & $975 / 32 / 0$ & $D$ & D \\
\hline Chr17:78354738 & exonic & missense & C. $13748 G>A$ & p.R4583Q & rs199887580 & $249 / 1 / 0$ & 208/0/0 & 1007/0/0 & $\mathrm{D}$ & D \\
\hline Chr17:78355494 & exonic & missense & c. 13945 C > G & p.L4649V & rs61745599 & 249/1/0 & 208/0/0 & 1007/0/0 & $\mathrm{D}$ & $\mathrm{D}$ \\
\hline Chr17:78356830 & exonic & missense & c. $14030 \mathrm{G}>\mathrm{T}$ & p.W4677 L & rs61741961 & 249/1/0 & 208/0/0 & 1006/1/0 & $\mathrm{D}$ & $\mathrm{D}$ \\
\hline Chr17:78357541 & exonic & missense & c. $14135 \mathrm{~A}>\mathrm{T}$ & p.N47121 & - & $249 / 1 / 0$ & 208/0/0 & $1007 / 0 / 0$ & $D$ & $D$ \\
\hline Chr17:78360656 & exonic & missense & c. $14887 \mathrm{C}>\mathrm{T}$ & p.R4963C & rs772035323 & $249 / 1 / 0$ & 208/0/0 & 1007/0/0 & $\mathrm{D}$ & $D$ \\
\hline Chr17:78362497 & splicing & - & c. $15000+8 C>T$ & - & - & $249 / 1 / 0$ & $208 / 0 / 0$ & $1006 / 1 / 0$ & - & - \\
\hline Chr17:78363181 & splicing & - & $\begin{array}{l}\text { C.15195+14C> } \\
T\end{array}$ & - & rs373144473 & $249 / 1 / 0$ & 208/0/0 & $1007 / 0 / 0$ & - & - \\
\hline
\end{tabular}

${ }^{\mathrm{a}}$ Genbank accession number: NM_001256071; ${ }^{\mathrm{b}}$ Genotype presented as wild type/heterozygous/hommozygous

c SIFT Score Prediction: $\mathrm{D}=$ damaging, $\mathrm{T}=$ tolerated; $\mathrm{c}$ POLYPHEN Score Prediction: $\mathrm{B}=$ benign, $\mathrm{P}=$ possibly damaging, $\mathrm{D}=$ probably damaging

- Not available 
Table 4 Single variant test and cumulative allele odds ratio of RNF213 rare variants

\begin{tabular}{|c|c|c|c|c|c|c|c|c|c|}
\hline Variant & Ref & Alt & Case_Ref & Case_Alt & Control_Ref & Control_Alt & OR & $95 \% \mathrm{Cl}$ & $p$ value \\
\hline Chr17:78247076-78,247,076 & $C$ & - & 499 & 1 & 2430 & 0 & - & - & 0.171 \\
\hline Chr17:78298891 & $\mathrm{T}$ & C & 499 & 1 & 2430 & 0 & - & - & 0.171 \\
\hline Chr17:78307986 & G & $\mathrm{T}$ & 499 & 1 & 2430 & 0 & - & - & 0.171 \\
\hline Chr17:78311532 & C & $\mathrm{T}$ & 498 & 2 & 2419 & 11 & 0.88 & $(0.20-4.00)$ & 1.000 \\
\hline Chr17:78311620 & A & G & 499 & 1 & 2430 & 0 & - & - & 0.171 \\
\hline Chr17:78313764 & C & $\mathrm{T}$ & 494 & 6 & 2414 & 16 & 1.83 & $(0.71-4.71)$ & 0.248 \\
\hline Chr17:78318465 & C & G & 496 & 4 & 2403 & 27 & 0.72 & $(0.25-2.06)$ & 0.383 \\
\hline Chr17:78319385 & $\mathrm{T}$ & G & 494 & 6 & 2408 & 22 & 1.33 & $(0.54-3.30)$ & 0.716 \\
\hline Chr17:78326772 & C & $\mathrm{T}$ & 499 & 1 & 2429 & 1 & 4.87 & $(0.30-77.95)$ & 0.312 \\
\hline Chr17:78350088 & $\mathrm{T}$ & C & 489 & 11 & 2392 & 38 & 1.42 & $(0.72-2.79)$ & 0.312 \\
\hline Chr17:78353469 & $\mathrm{T}$ & C & 499 & 1 & 2430 & 0 & - & - & 0.171 \\
\hline Chr17:78354738 & G & A & 499 & 1 & 2430 & 0 & - & - & 0.171 \\
\hline Chr17:78355494 & C & G & 499 & 1 & 2429 & 1 & 4.87 & $(0.30-77.95)$ & 0.312 \\
\hline Chr17:78356830 & G & $\mathrm{T}$ & 499 & 1 & 2430 & 0 & - & - & 0.171 \\
\hline Chr17:78357541 & A & $\mathrm{T}$ & 499 & 1 & 2430 & 0 & - & - & 0.171 \\
\hline Chr17:78360656 & C & $\mathrm{T}$ & 499 & 1 & 2429 & 1 & 4.87 & $(0.30-77.95)$ & 0.312 \\
\hline Chr17:78362497 & C & $\mathrm{T}$ & 499 & 1 & 2430 & 0 & - & - & 0.171 \\
\hline Chr17:78363181 & C & $\mathrm{T}$ & 499 & 1 & 2430 & 0 & - & - & 0.171 \\
\hline Total counts & & & 8958 & 42 & 43,623 & 117 & 1.74 & $(1.23-2.49)$ & 0.002 \\
\hline
\end{tabular}

${ }^{*} P$ values were calculated using a two-sided Fisher's exact test

Ref Reference allele(s), Alt Alternate allele(s)

Case $=250$ ICASO patients, Control $=1 \mathrm{KG}$ Chinese Han population and GeneSky Database(including 1007 healthy individuals)

were specific in Chinese MMD except p.D4013N (found in Caucasian) and p.R4062Q (found in Caucasian and Japanese) [12, 14, 18, 21, 26]. Moreover, p.D4863N, p.E4950D and p.A5021V were particularly identified among Chinese MMD patients in several studies [12, 14, 21]. In non-East Asian countries, numerous RNF213 variants were identified. Cecci et al. identified 10 variants only in Caucasian MMD patients [27] and Shoemaker et al. identified 22 variants in multiple non-East Asian population [26] (Listed in Additional file 2: Table S1). Whether these rare variants or other RNF213 rare variants associated with ICASO like p.R4810K or not is unclear currently. In this study, we performed target exome sequencing of RNF213 in ICASO patients and found 18 rare variants in 39 patients. All the 18 rare variants identified were novel and not have been found in previous MMD studies. This result suggested that RNF213 rare variants spectrum of Chinese ICASO is distinct from MMD, locus heterogeneity of RNF213 is highly indicated between ICASO and MMD.

Recent studies suggested that RNF213 genotypes were associated with MMD phenotypes, numerous studies confirmed that the homozygous p.R4810K variant carriers present earlier onset age, severe symptoms and prognosis $[13,17,28]$. Zhang et al. demonstrated Chinese MMD patients with RNF213 p.R4810K were younger at diagnosis than those without $R N F 213$ rare variants
(25 vs 29 years old, $p=0.049$ ), more ischemic cases and more likely to occur at posterior cerebral artery [21]. In addition, researchers also identified the association of RNF213 variants and ICASO phenotypes. Bang et al. indicated that Korean ICASO patients with RNF213 p.R4810K variant were younger at diagnosis than those without the variant $(52.6 \pm 9.6$ vs $56.8 \pm 12.7$ years old, $p=0.027$ ) [6]. But our study showed that mean age at diagnosis of RNF213 rare variants carriers was older than that of non-carriers. Considering the complicated current medical status of China, it is difficult to confirm the precise onset age during the slow progression of ICASO unless the patients seek a medical treatment at the early stage of ICASO (such as recurrent headache, dizziness and transient ischemic attack). The majority of patients $(66.6 \%)$ in our study suffered from severe acute cerebral infarction as initial clinical symptoms, so the age at diagnosis in our research may not be able to reflect the true onset age of ICASO.

In our study, the presence of anterior and posterior ICASO showed no significant difference between RNF213 rare variants carriers and non-carriers. This result is consistent with Bang's study. They reported that the presence of proximal carotid artery and posterior ICASO showed no significant difference between RNF213 p.R4810K carriers and non-carriers [6]. But in 
Table 5 Characteristics of RNF213 rare variants carriers and non-carriers

\begin{tabular}{|c|c|c|c|}
\hline Characteristics & Carrier $(n=39)$ & Non-carrier $(n=211)$ & $p$ value \\
\hline \multicolumn{4}{|l|}{ Age(years) } \\
\hline Mean \pm SD & $60.5 \pm 6.2$ & $57.3 \pm 8.9$ & 0.028 \\
\hline Female, n(\%) & 12(30.8) & 65(30.8) & 0.996 \\
\hline \multicolumn{4}{|l|}{ Risk factors, n(\%) } \\
\hline Smoking & $16(41.0)$ & $81(38.4)$ & 0.756 \\
\hline Drinking & 14(35.9) & $49(23.2)$ & 0.095 \\
\hline Hypertension & $29(74.4)$ & 123(58.3) & 0.059 \\
\hline Diabetes & 12(30.8) & $48(22.7)$ & 0.281 \\
\hline Hyperlipemia & 14(35.9) & $81(38.4)$ & 0.768 \\
\hline \multicolumn{4}{|l|}{ Clinical symptoms, n(\%) } \\
\hline Hemorrhage & $3(7.7)$ & $4(1.9)$ & 0.137 \\
\hline Infarction & $29(74.4)$ & $136(64.5)$ & 0.23 \\
\hline Ischemia & $8(20.5)$ & $69(32.7)$ & 0.13 \\
\hline Other & $1(2.6)$ & $8(3.8)$ & \\
\hline \multicolumn{4}{|c|}{ Characteristics of cerebral artery stenosis, n(\%) } \\
\hline Multiple stenosis & $29(74.4)$ & 170(80.6) & 0.377 \\
\hline \multicolumn{4}{|l|}{ Location } \\
\hline Anterior circulation ${ }^{a}$ & $4(10.3)$ & $32(15.2)$ & 0.688 \\
\hline Posterior circulation ${ }^{b}$ & 14(35.9) & $85(40.3)$ & \\
\hline Multiple circulation ${ }^{c}$ & 20(51.3) & $91(43.1)$ & \\
\hline Others & $1(2.6)$ & $3(1.4)$ & \\
\hline
\end{tabular}

${ }^{\mathrm{a}}$ Anterior circulation $=A C A$ Anterior cerebral artery, MCA Middle cerebral artery, ICA Internal carotid artery

${ }^{\mathrm{b}}$ Posterior circulation $=B A$ Basilar artery, VA Vertebral artery, $P C A$ Posterior cerebral artery

${ }^{c}$ Multiple circulation = including both Anterior and posterior circulation

Shinya's study, all of 10 RNF213 p.R4810K carriers suffered anterior ICASO and no one suffered posterior ICASO [10], they defined the posterior circulation as the basilar artery and vertebral artery, patients with posterior cerebral artery stenosis were not found in their case series. The difference of patient selection may explain the discrepancy.

ICASO is caused by environmental and genetic risk factors. Though some specific rare variants of RNF213 were identified in Chinese ICASO patients, but there were no specific clinical characteristics of the RNF213 rare variants carriers, which illustrating that rather than RNF213 variants there may be other risk factors of ICASO in Chinese. Studies focus on multiple factors including genetic and environmental are needed.

The limitation of this study should be mentioned. First, the sample size of our study was relatively small and the strictly matched cerebrovascular disease-free controls were not available; the reference allele frequencies of rare variants in unaffected individuals were obtained from 1000 Genomes Project and Genesky in-house database. Second, atherosclerosis is the most common cause of ICASO, it usually caused by acquired factors, such as hypertension, diabetes mellitus, dyslipidemia, and smoking. However, these information were not available in the control databases, so we cannot take these environmental risk factors as covariate when we implemented the gene-based association test.

\section{Conclusion}

Rare variants of RNF213 are associated with ICASO in Chinese, but there were no specific clinical characteristics of the RNF213 rare variants carriers. Genetic diagnosis values for this gene among Chinese ICASO patients are limited. Rather than RNF213 variants there should be other risk factors of ICASO in Chinese. Studies focus on multiple factors including genetic and environmental are needed.

\section{Additional files}

Additional file 1: Figure S1. RNF213 variants identified in MMD and ICASO patients around the world. (TIF $712 \mathrm{~kb}$ )

Additional file 2: Table S1. Rare variants of RNF213 identified in worldwide MMD patients. (XLSX $14 \mathrm{~kb}$ )

\begin{abstract}
Abbreviations
ACA: Anterior cerebral artery; BA: Basilar artery; BWA: Burrows-Wheeler Aligner; GATK: Genome Analysis Toolkit; ICA: Internal carotid artery; ICASO: Non-moyamoya intracranial major artery stenosis/occlusion; MAF: Minor allele Frequency; MCA: Middle cerebral artery; MMD: Moyamoya disease; PCA: Posterior cerebral artery; PolyPhen-2: Polymorphism Phenotyping V2; RNF213: The ring finger protein 213; SIFT: Sorting Intolerant From Tolerant; SKAT: Sequence Kernel Association Test; SKAT-O: SKAToptimal test; VA: Vertebral artery; WSS: Weight Sum Statistic
\end{abstract}

\section{Acknowledgements}

We thank all participants for providing samples and also those involved in the sample collection.

\section{Funding}

This work was supported by grants from the National Nature Science Foundation of China, Nature Science Foundation of Hunan province and China postdoctoral science foundation to J.Y (No. 81502881, 2017JJ3428, and 2015 M582351). Dr. Yan is a postdoctoral fellow at Central South University (No. 149946), supported by the Postdoctoral International Exchange Plan in China. XL is a postgraduate student of Central South University, supported by the fundamental research funds for the Central Universities of Central South University (No.2018zzts851). The funding bodies did not involve in the design of the study, collection, analysis, interpretation of data and writing of the manuscript.

\section{Availability of data and materials}

The data that support the findings of this study are available from Genesky Bio-Tech Company but restrictions apply to the availability of these data, which were used under license for the current study, and so are not publicly available. Data are however available from the authors upon reasonable request and with permission of Genesky Bio-Tech Company.

\section{Authors' contributions}

TZ, BL participated in the patient recruitment, sample collection and clinical data explanation. $\mathrm{XL}, \mathrm{SH}, J \mathrm{~L}, J \mathrm{~J}$ and $J \mathrm{Y}$ carried out the experiments and data analysis. $\mathrm{XL}, \mathrm{HT}$ and JY took charge of study design, coordination and drafted the manuscript. All authors read and approved the final manuscript. 


\section{Ethics approval and consent to participate}

This study was approved by the Medical Ethics Committee of Central South University and the survey participants gave informed consent before the interview and blood samples were taken.

\section{Consent for publication}

Not applicable.

\section{Competing interests}

The authors declare that they have no competing interests.

\section{Publisher's Note}

Springer Nature remains neutral with regard to jurisdictional claims in published maps and institutional affiliations.

\section{Author details}

'Department of Epidemiology and Health Statistics, XiangYa School of Public Health, Central South University, Changsha 410078, Hunan, China. ${ }^{2}$ Department of Neurology, the Second Hospital of Hebei Medical University, Heping West Road, Xinhua District, Shijiazhuang 050000, Hebei, China. ${ }^{3}$ Department of Neurosurgery, XiangYa Hospital, Central South University, 87 Xiangya Road, Changsha 410008, Hunan, China.

Received: 10 January 2019 Accepted: 18 March 2019

Published online: 29 March 2019

\section{References}

1. Suri MF, Qiao Y, Ma X, Guallar E, Zhou J, Zhang Y, et al. Prevalence of intracranial atherosclerotic stenosis using high-resolution magnetic resonance angiography in the general population: the atherosclerosis risk in communities study. Stroke. 2016;47(5):1187-93.

2. Wong LK. Global burden of intracranial atherosclerosis. Int J Stroke. 2006; 1(3):158-9.

3. Miyawaki S, Imai H, Shimizu M, Yagi S, Ono H, Mukasa A, et al. Genetic variant RNF213 c.14576G >a in various phenotypes of intracranial major artery stenosis/occlusion. Stroke. 2013;44(10):2894-7.

4. Miyawaki S, Imai H, Takayanagi S, Mukasa A, Nakatomi H, Saito N. Identification of a genetic variant common to moyamoya disease and intracranial major artery stenosis/occlusion. Stroke. 2012;43(12):3371-4.

5. Bang OY, Ryoo S, Kim SJ, Yoon CH, Cha J, Yeon JY, et al. Adult Moyamoya disease: a burden of intracranial stenosis in east Asians? PLoS One. 2015; 10(6):e0130663.

6. Bang OY, Chung JW, Cha J, Lee MJ, Yeon JY, Ki CS, et al. A polymorphism in RNF213 is a susceptibility gene for intracranial atherosclerosis. PLoS One. 2016;11(6):e0156607

7. Kim YJ, Lee JK, Ahn SH, Kim BJ, Kang DW, Kim JS, et al. Nonatheroscleotic isolated middle cerebral artery disease may be early manifestation of Moyamoya disease. Stroke. 2016:47(9):2229-35.

8. Park YS, An HJ, Kim JO, Kim WS, Han IB, Kim OJ, et al. The role of RNF213 4810G $>a$ and 4950G $>a$ variants in patients with Moyamoya disease in Korea. Int J Mol Sci. 2017;18(11). https://doi.org/10.3390/ijms18112477.

9. Zhang T, Guo C, Liao X, Xia J, Wang X, Deng J, et al. Genetic analysis of RNF213 p.R4810K variant in non-moyamoya intracranial artery stenosis/ occlusion disease in a Chinese population. Environ Health Prev Med. 2017; 22(1):41.

10. Shinya $\mathrm{Y}$, Miyawaki S, Imai H, Hongo H, Ono H, Takenobu A, et al. Genetic analysis of ring finger protein 213 (RNF213) c.14576G>a in intracranial atherosclerosis of the anterior and posterior circulations. J Stroke Cerebrovasc Dis. 2017:26(11):2638-44.

11. Kamada F, Aoki Y, Narisawa A, Abe Y, Komatsuzaki S, Kikuchi A, et al. A genome-wide association study identifies RNF213 as the first Moyamoya disease gene. J Hum Genet. 2011;56(1):34-40.

12. Liu W, Morito D, Takashima S, Mineharu Y, Kobayashi H, Hitomi T, et al. Identification of RNF213 as a susceptibility gene for moyamoya disease and its possible role in vascular development. PLoS One. 2011;6(7):e22542.

13. Miyatake S, Miyake N, Touho H, Nishimura-Tadaki A, Kondo Y, Okada I, et al. Homozygous C.14576G>a variant of RNF213 predicts early-onset and severe form of moyamoya disease. Neurology. 2012;78(11):803-10.

14. Wu Z, Jiang $H$, Zhang $L$, Xu X, Zhang $X$, Kang Z, et al. Molecular analysis of RNF213 gene for moyamoya disease in the Chinese Han population. PLoS One. 2012;7(10):e48179.
15. Wang $X$, Zhang $Z$, Liu W, Xiong $Y$, Sun W, Huang $X$, et al. Impacts and interactions of PDGFRB, MMP-3, TIMP-2, and RNF213 polymorphisms on the risk of Moyamoya disease in Han Chinese human subjects. Gene. 2013; 526(2):437-42.

16. Bang OY. Intracranial atherosclerosis: current understanding and perspectives. J Stroke. 2014;16(1):27-35

17. Kim EH, Yum MS, Ra YS, Park JB, Ahn JS, Kim GH, et al. Importance of RNF213 polymorphism on clinical features and long-term outcome in moyamoya disease. J Neurosurg. 2015;124(5):1221-7.

18. Lee MJ, Chen YF, Fan PC, Wang KC, Wang K, Wang J, et al. Mutation genotypes of RNF213 gene from moyamoya patients in Taiwan. J Neurol Sci. 2015;353(1-2):161-5.

19. Huang Y, Cheng D, Zhang J, Zhao W. Association between RNF213 gene polymorphisms and the genetic susceptibility of adult moyamoya disease of Zhuang population in Guangxi. J Apoplexy Nerv Dis. 2015:32(10):918-21.

20. Xue S, Cheng W, Wang W, Song H, Feng W, Ovbiagele B. Genetic variant RNF213 in non-MMD intracranial major artery stenosis/occlusion in Chinese Han population and HR-MRI findings. Stroke. 2017;48(Suppl 1):AWP151.

21. Zhang Q, Liu Y, Zhang D, Wang R, Zhang Y, Wang S, et al. RNF213 as the major susceptibility gene for Chinese patients with moyamoya disease and its clinical relevance. J Neurosurg. 2017;126(4):1106-13.

22. Liao X, Deng J, Dai W, Zhang T, Yan J. Rare variants of RNF213 and moyamoya/non-moyamoya intracranial artery stenosis/occlusion disease risk: a meta-analysis and systematic review. Environ Health Prev Med. 2017; 22(1):75.

23. Koizumi A, Kobayashi $\mathrm{H}$, Hitomi T, Harada KH, Habu T, Youssefian S. A new horizon of moyamoya disease and associated health risks explored through RNF213. Environ Health Prev Med. 2016;21(2):55-70.

24. Madsen BE, Browning SR. A groupwise association test for rare mutations using a weighted sum statistic. PLoS Genet. 2009;5(2):e1000384.

25. Moteki $Y$, Onda $H$, Kasuya $H$, Yoneyama $T$, Okada $Y$, Hirota $K$, et al. Systematic validation of RNF213 coding variants in Japanese patients with Moyamoya disease. J Am Heart Assoc. 2015;4(5). https://doi.org/10.1161/ JAHA.115.001862.

26. Shoemaker LD, Clark MJ, Patwardhan A, Chandratillake G, Garcia S, Chen R, et al. Disease variant landscape of a large multiethnic population of Moyamoya patients by exome sequencing. G3 (Bethesda, Md). 2015;6(1):41-9.

27. Cecchi AC, Guo D, Ren Z, Flynn K, Santos-Cortez RL, Leal SM, et al. RNF213 rare variants in an ethnically diverse population with Moyamoya disease. Stroke. 2014:45(11):3200-7.

28. Miyatake S, Touho H, Miyake N, Ohba C, Doi H, Saitsu H, et al. Sibling cases of moyamoya disease having homozygous and heterozygous C.14576G>a variant in RNF213 showed varying clinical course and severity. J Hum Genet. 2012:57(12):804-6.

Ready to submit your research? Choose BMC and benefit from:

- fast, convenient online submission

- thorough peer review by experienced researchers in your field

- rapid publication on acceptance

- support for research data, including large and complex data types

- gold Open Access which fosters wider collaboration and increased citations

- maximum visibility for your research: over $100 \mathrm{M}$ website views per year

At BMC, research is always in progress.

Learn more biomedcentral.com/submissions 